\title{
Solutions to the Three-Phase Buckley-Leverett Problem
}

\author{
GUZMAN, Rafael E., Stanford University/ now BPX Colombia \\ FAYERS, F. John, Stanford University/now consultant
}

Paper presented at the 5th European Conference on the Mathematics of Oil Recovery, Leoben, Austria, 3-6 Sept. 1996

\begin{abstract}
This paper presents method of characteristics (MOC) solutions to the three-phase Buckley-Leverett problem with and without gravity, for the three classes of equations described by Guzmán and Fayers (1996). Thus, solutions for equations with single and multiple umbilic points, and mixed elliptic and hyperbolic equations are constructed. A close relation between the mathematical properties of the equations (in terms of location and quantity of umbilic points) and the physics of flow is described. This relationship can be observed by the effect of gravity on the efficiency of the displacement, the formation of oil and water banks, and the presence of a unique or infinite solution paths within the three-phase flow region for a class of solutions. Elliptic regions did not generate physically inadmissible effects and stable shocks with saturations on opposite sides of elliptic regions were constructed.
\end{abstract}

\section{INTRODUCTION}

Three-phase flow in porous media is a fundamental constituent of many oil recovery processes. Despite its common occurrence and relevance, our mathematical and physical understanding of three-phase flow is not satisfactory. This paper is the second part of a study on the mathematical theory of three-phase flow. The problem considered is the extension of the classical twophase Buckley-Leverett (1941) theory to the flow of three phases.

Despite the necessary simplifying assumptions, the Buckley-Leverett theory has been one of the most useful techniques in reservoir engineering. Although the theory may be more suitahle for laboratory settings (Grader and O'Meara, 1988), the extension to three-phase flow can provide information to better interpret full three dimensional simulations, test problems and new methods for numerical simulators, insight into the physics of displacements, and methods to interpret laboratory flow experiments.

Solutions for three-phase flow problems that assume Corey-type relative permeabilities and no gravity have been presented by many authors (Refs. 26, 17, 1, 5, and
6). Full solutions and a complete mathematical theory was presented by Isaacson et al. (1988). Bell et el. (1986) used finite differences to compute solutions with Stone-type relative permeabilities and to investigate the effect of the elliptic regions. Satisfactory solutions were found even when either the injection or initial conditions were inside an elliptic region. Example solutions were constructed using the method of characteristics by Gorell (1988) and Wingard and Orr (1994). Gorell (1988) ignored the imaginary part of the complex eigenvalues without a mathematical justification. Wingard and Orr (1994) included mass transfer and temperature effects, and the issues of threephase flow were not presented in a systematic way.

Solutions for problems using Corey-type relative permeabilities made a significant contribution to the understanding of three-phase flow displacements. However, these solutions are not able to explain the three-phase flow experimental results of Grader and O'Meara (1988). Contrary to the observed behavior, solutions constructed using a Corey-type model would predict the same saturation path inside the three-phase region (Falls and Schulte, 1992b). Sahni et al. (1996) used the theory in this paper to interpreted the experiments of Grader and O'Meara (1988): More elaborated relative permeability models were necessary to obtain good agreement between analytic and experimental recovery curves and saturation paths. In addition, the analytic theory in this paper was also used by Sahni et al. (1996) to verify the extension to the Johnson, Bossler, and Naumann (1959) (JBN) analysis to interpret dynamic two-phase relative permeability measurements, to three-phase measurements presented by Virnovsky (1984) and Grader and O'Meara (1988).

This paper presents solutions for three-phase flow problems with and without gravity, using Corey, extended Corey, and Stone-type models. Practical conditions are simulated by using measured two-phase flow data and Stone's model I (Stone, 1970), and reasonable viscosity ratios and gravity numbers. It is shown that the structure of the new solutions is very different from the previous solutions to unique umbilic point problems (Refs. 26, 17, 1, 5, and 6) (i.e., using Corey-k $\mathbf{k}_{\mathbf{r}}$. 


\section{METHOD OF SOLUTION}

Mathematically, the three-phase Buckley-Leverett problem is the well known Riemann problem. The Riemann problem consists of the conservation laws (see Guzmán and Fayers (1996) for a definition of all the terms in the equations)

$$
s_{t}+f_{x}=s_{t}+A s_{x}=0
$$

inside the three-phase flow domain, for $-\infty<\mathrm{x}<\infty, \mathrm{t}$ $>0$, with the initial condition

$$
s(x, 0)=\left\{\begin{array}{l}
s_{r}, x<0 \\
s_{l}, x>0
\end{array}\right.
$$

Solutions to Riemann problems are well known to be solely functions of $\eta=x / t$ (i.e., $s$ is constant along $\eta$ characteristics (Lax, 1957)). Applying the selfsimilarity transformation $\eta=x / t$ to Eq. (1) gives the system of ordinary differential equations

$$
(\eta I-A) d s=0
$$

Eq. (3) is an eigenvalue problem with $\eta=x / t$ as the eigenvalues of matrix $A$ and $d s$ as the right eigenvectors. The eigenvalues are given by

$$
\eta=1 / 2\left[\left(f_{w w}+f_{R g}\right) \pm \sqrt{\delta}\right]
$$

and the right eigenvectors given by

$$
d s=\left(\begin{array}{c}
1 \\
d S_{g} / d S_{w}
\end{array}\right)=\left(\begin{array}{c}
1 \\
\left(f_{g g}-f_{w w} \pm \sqrt{\delta}\right) / 2 f_{w g}
\end{array}\right)
$$

where $\delta=\left(f_{w w}-f_{g g}\right)^{2}+4 f_{g w} f_{w g}$ is the discriminant.

The Riemann problem is reduced to connecting two states ( $s_{l}$ and $s_{r}$ ), with waves moving at the wave or characteristic velocity $\eta$, along curves with direction given by the eigenvectors $d s$. These curves are called rarefaction curves, and are obtained by integration of $\mathrm{dS}_{\mathrm{g}} / \mathrm{dS} \mathrm{W}_{\mathrm{W}}$ from Eq. (5) (see Falls and Schulte (1992b) for a recommended procedure). Solutions are composed of constant states, rarefaction waves, and shock waves. For constant states, $s$ remains constant while $\mathrm{x} / \mathrm{t}$ increases. Constant states separate shocks that have different velocities and rarefaction waves that belong to a different family of eigenvalues. Rarefaction waves are continuous solutions along a rarefaction curve with monotonically increasing eigenvalue from left to right. Shock waves are discontinuities in the saturation profiles ( $d s / d \eta \rightarrow \infty$ ). They do not satisfy Eq. (3) but a related integral equation (LeVeque, 1992).
Shock waves are admissible weak solutions, i.e., solutions that satisfy the related integral equation (Oleinik, 1957), if they satisfy the Rankine-Hugoniot or jump condition and the entropy condition. The Rankine-Hugoniot condition is simply. an integral balance of the quantity $s$ across the shock. For a system of $n$ conservation laws it is given by (Lax, 1957)

$$
\sigma\left[S_{k}\right]=\left[f_{k}\right], k=1, \cdots, n
$$

for some value of the shock velocity $\sigma$. The symbol [] denotes the jump of the enclosed quantity. Entropy conditions are in general obtained by taking the zero diffusion limit of a diffusive profile (Oleinik, 1957). For genuinely nonlinear ${ }^{1}$ and strictly hyperbolic problems, Lax (1957) introduced the necessary entropy conditions by requiring that $n+1$ characteristics impinged or run into the shock (producing a stable shock). Liu (1975) extended Lax's entropy conditions to systems that are not necessarily genuinely nonlinear (but still strictly hyperbolic). Liu's entropy conditions state that

$$
\sigma \leq \frac{f_{i}\left(s_{i}\right)-f_{i}\left(s_{i-}\right)}{s_{i}-s_{i-}}
$$

for any state $s$ between $s_{\text {. }}$ and $s_{+}$.

Conservation laws that are not strictly hyperbolic can give rise to a new type of waves (Isaacson et al., 1989) called transitional waves. These waves (rarefactions and/or shock waves) are a direct consequence of the presence of umbilic points (Refs. 18,17, and 8). Transitional waves were, however, not observed in the examples used in this work due to the selected initial conditions. Nonetheless, they become important for certain initial conditions (Isaacson et al. 1988).

\section{ON THE CORRECT FORMULATION OF THE RIEMANN PROBLEM}

Liu (1975) has shown existence and uniqueness of the solution to Riemann problems for systems of strictly hyperbolic conservation laws, and proposed the entropy condition of Eq. (7). Existence and uniqueness for systems of equations that are not strictly hyperbolic is still an open question (Refs. 8, 18). The standard theory of allowing only shocks that are zero diffusion limits of diffusive solutions may not provide a unique solution (Refs. 14, 16, and 2) and additional stronger conditions may be needed. On the other hand, allowing only Lax and Liu shocks may prove to be insufficient to guarantee existence of solutions (Isaacson et al., 1989).

\footnotetext{
${ }^{1}$ The system of Eq. (1) is said to be genuinely nonlinear if each eigenvalue $\eta_{i}$ changes in a strictly . monotonic manner along every rarefaction curve of its own family (LeVeque, 1992), i.e., if $d s_{i} \cdot \nabla \eta_{i} \neq 0$.
} 
The conservation equations for three-phase flow can have isolated or multiple umbilic points or elliptic regions where strict hyperbolicity fails. Thus, from the preceding discussion, it has not been established absolutely whether the Riemann problem for threephase flow is well posed. This subject is beyond the scope of this paper and will not be addressed further. For the example solutions presented in the coming sections, existence is shown by direct construction of a solution, continuity is shown by variation of initial data, and uniqueness is indicated by the satisfaction of entropy conditions, and by comparison with numerical evidence. Only problems with both left and right states outside the elliptic regions are considered.

\section{SOLUTIONS TO PROBLEMS WITH COREY $\mathbf{K}_{\underline{\mathbf{r}}}$}

Solutions to the three-phase flow problem without gravity and Corey $\mathbf{k}_{\mathbf{r}}$ (unique umbilic point equations (Guzmán and Fayers, 1996)) have been presented by many authors (Refs. 26, 17, 1, 5, and 6). The full Riemann solution for this problem was constructed by Isaacson et al. (1988) using the concept of wave boundaries. The solutions constructed in this paper follow the procedure of Isaacson et al. (1988). The MOC solutions are compared with numerical explicit single point upstream (SPU) finite differences (FD) solutions (LeVeque, 1992). The agreement between the FD and MOC solutions was usually excellent. This was achieved by properly selecting a stable time step, using between 1000 to 4000 grid blocks, and calculating the solutions up to $t=0.5$. The shocks for the MOC solutions are represented in the saturation ternary diagram by upstream and downstream points only, whereas, they are continuous variations for the FD solutions. All the following solution figures include both the numerical and the MOC solution. Due to the excellent agreement, it is sometimes hard to see any difference (specially in the saturation profiles) between the two solutions.

Fig. 1 shows the solutions for a system with $\lambda_{i}=S_{i}^{2}$, $s_{l}=(0.9,0.1)$ and $s_{r}=(0.2,0)$. The solution starts at the left state a, and follows a 1-rarefaction curve up to point $b$. The 1-rarefaction is represented as a continuous variation in both the saturation space and the saturation profiles. At point $b$ there is a constant state in which the solution changes from a 1-rarefaction wave to a faster 2-rarefaction wave. This constant state is shown as a sharp change of slope in the profiles. Note that the limit of the first 1 -wave corresponds to a point on the inflection locus, i.e., it occurs when the 1-wave attains its maximum velocity. The solution proceeds from point $b$ to point $c$ on a 2-rarefaction wave which necessarily passes through the umbilic point (Falls and Schulte, 1992b). The water saturation profile clearly shows this rarefaction as an increase in water saturation. The limit of the 2-rarefaction wave (point c) is a point on the 2-interior contact locus (i.e., the upstream state of a Buckley-Leverett shock). From point $\mathrm{c}$ the solution has a Buckley-Leverett shock to point $d$ on the two phase line where $S_{g}=0$. The solution from points b to d belongs to the same 2-wave group. Once the solution is on the two-phase region, it proceeds as in the classical Buckley-Leverett theory. For this example, there is a constant state followed by a genuine shock (i.e.; a shock with velocity different from the characteristics velocities upstream and downstream of the shock) from point $\mathbf{d}$ to $\mathrm{e}$ where the solution arrives to the right state e. The constant state at $\mathbf{d}$ is needed since the shock velocity in the two-phase region is faster than the shock in the three-phase region.

The three unknowns to construct the Buckley-Leverett shock from $\mathrm{c}$ to $\mathrm{d}$ (i.e., the two saturations at $\mathrm{c}$ and the water saturation at d) are obtained using the equations for the Rankine-Hugoniot condition, the entropy condition, and the relationship between $S_{W}$ and $S_{g}$ on the 2-rarefaction (three equations). The equation for the Rankine-Hugoniot condition is given by (obtained from Eq. 6)

$$
\frac{\left[f_{w}\right]\left[S_{g}\right]}{\left[f_{g}\right]\left[S_{w}\right]}-1=0
$$

The entropy condition in this case is equivalent to setting the shock velocity equal to the 2-eigenvalue at point c, i.e.,

$$
\frac{\left[f_{g}\right]}{\left[s_{g}\right]}=\eta_{2}\left(s_{+}\right)
$$

The third relationship is obtained from step by step integration along the 2 -family rarefaction curve that starts at point $b$.

A very interesting fact about these solutions was recognized by Falls and Schulte (1992b). The solution in Fig. 1 will be identical inside the three-phase region even after drastically changing the initial right state (e.g., from $s_{r}=(0.2,0)$ to $\left.s_{r}=(0.7,0)\right)$. The reason for this behavior is the necessity of following the 2 rarefaction curve that passes through the unique umbilic point. This remarkable behavior is caused by the selection of Corey-type relative permeabilities and the presence of a unique umbilic point. For more general relative permeability models (observed in the laboratory), this behavior does not occur, as shown later.

\section{SOLUTIONS TO PROBLEMS WITH EXTENDED COREY $K_{\mathbf{r}}$}

The phase mobilities $\lambda_{w}=S_{w}^{2} /\left(S_{w}^{2}+\left(1-S_{w}\right)^{2}\right)$, $\lambda_{g}=10 S_{g}^{2}$, and $\lambda_{o}=-2 S_{o}^{3}+3 S_{o}^{2}$, used to show the existence of multiple umbilic points (Guzmán and Fayers, 1996), will now be used to construct example solutions to the three-phase Riemann problem. 


\section{Displacements Without Gravity}

The resulting rarefaction curves for the above model are shown in Fig. 2. The two singular umbilic points are easily recognized by the congruence of more than two rarefaction curves. The fan of 2-rarefaction curves between the two umbilic points of Fig. 2. is significantly different from the one for a single umbilic point (Refs. 27 and 5). This difference will play a very important role in the solutions as shown later. As in the single umbilic point, the rarefactions passing through the umbilic points serve as asymptotes for both families. The three comers of the ternary diagram are still singular points with an infinite number of 2 rarefactions passing through them. There is a rarefaction curve of each family joining the two umbilic points.

Fig. 3 shows four example solutions to problems with the same left state $s_{l}=(0.1,0.9)$ but different right states. The right state for each solution is clear from Fig. 3. Saturation profiles for the solution with $s_{r}=$ $(0.5,0)$ is shown in Fig. 4. All the solutions are composed of a 1-rarefaction followed by a constant state, and a 2-rarefaction followed by a 2-BuckleyLeverett shock to the right state. Each of these solutions is constructed following the procedure outlined for the solution in Fig. 1. Unlike the solutions for single umbilic point problems, each solution follows a different path in the three-phase region. The necessity of following a unique path inside the threephase region for the unique umbilic point problems was due to the presence of a special path through the umbilic point (Falls and Schulte, 1992b). The presence of a fan of 2-rarefaction curves for the two-umbilic point model prevents that from happening. Now each solution picks a different 2-rarefaction depending on where the right state is located. The unique path inside the three-phase region for the solutions of Falls and Schulte (1992b) is therefore only a consequence of the selection of a particular relative permeability model and not a property of the solutions for three-phase flow in general.

Another difference between the solutions of unique and multiple umbilic points is shown in Fig. 4. The water bank in Fig. 1 is no longer present in Fig. 4. This difference is again due to the fan of 2-rarefactions present in the two umbilic point model. The solutions in Fig. 4 can directly shock to the right state in the two-phase $S_{W}-S_{0}$ line, avoiding the intermediate constant state and second shock present in the solution of Fig. 1.

\section{Displacements With Gravity}

Gravity numbers move the umbilic point(s) towards the maximum saturation of a phase if the efficiency of the displacement by that phase is increased (Guzmán and Fayers, 1996). For example, positive gravity numbers move the umbilic point towards maximum gas saturation and minimum water saturation and this indicates an increase in the efficiency of the displacement by gas and a decrease in the efficiency of the displacement by water.

Fig. 5 shows the solution to the same problem of Fig. 4 , but including gravity $\left(N_{g}=2\right.$ and $\left.N_{W}=1 / 2\right)$. The change in gravity numbers has also removed one of the umbilic points, leaving a unique umbilic point at $s=$ $(0.236,0.080)$. The solution from left to right starts with a 1-rarefaction between $\mathbf{a}$ and $\mathbf{b}$, there is a constant state at b, a 2-rarefaction from $b$ to c, a contact discontinuity or indifferent wave between $\mathbf{c}$ and $\mathbf{d}$, a 2 rarefaction from $d$ to $e$ and a Buckley-Leverett shock from e to the right state $f$. From point $d$ and on there is only one family of rarefactions since the solution is now in the two-phase region, but the rarefaction corresponds to a 2-rarefaction in the three-phase region. The solution from a to $\mathbf{d}$ is constructed as in the previous examples. From $\mathbf{d}$ to $\mathbf{f}$ the solution proceeds as in the classical Buckley-Leverett theory with a rarefaction from $\mathbf{d}$ to $e$ and a tangent shock from $e$ to $f$.

The effect of gravity can be seen by comparing the solutions in Figs. 4 and 5. A positive gas gravity number has improved the displacement by gas by decreasing the gas breakthrough time (smaller gas shock velocity), increasing the amount of oil recovered at gas breakthrough (larger gas shock height), and decreasing the size of rarefactions upstream of the gas shock (speed up in oil recovery). On the other hand, a positive water gravity number has decreased the efficiency of water displacement by creating a second water shock that breaks through earlier than the gas, and shortens the size of the oil bank created by the gas. Mathematically, these two effects are due to the removal of one of the umbilic points and the movement of the remaining one towards maximum gas saturation and minimum water saturation. The removal of one of the umbilic points makes the solution follow the 2-rarefaction that passes through the remaining umbilic point and creates the oil bank in the two-phase region.

Point $\mathbf{c}$ of Fig. 5 is the upstream state of an indifferent wave from $\mathbf{c}$ to $\mathbf{d}$, and therefore a point on the double contact locus. The reason for the presence of an indifferent wave in this solution is the reduction of velocity of point $\mathbf{c}$, and the increase of velocity for saturations on the two-phase line $S_{g}=0$, caused by gravity. Compared to the solution in Fig. 6 , point $c$ moves slow enough such that the solution can no longer shock directly to the initial condition (point $\mathbf{d}$ in Fig. 4 and point $f$ in Fig. 5). The solution in Fig. 5 shocks to a point on the $S_{g}=0$ line that has the same velocity as in point $\mathbf{c}$ (i.e., the indifferent wave between c and d), and then increases velocity following the rarefaction $d$ to $e$ and the shock from e to $f$. In other words, point $c$ in Fig. 4 has a velocity that is faster than any velocity on the $S_{g}=0$ line so that the solution must directly shock to the right state $d$. The movement of the umbilic point is responsible for the other changes in the observed behavior. This example demonstrates the importance of a careful analysis of the mathematical properties of the equations before studying the solutions to initial-value problems. 


\section{SOLUTIONS TO PROBLEMS WITH STONE $\underline{\mathbf{K}}_{\mathbf{r}}$}

The experimental two-phase data of Saraf and Fatt (1967) and the UKLB data set (Fayers, 1989), together with Stone's model I were used to construct the following solutions. As such, three-phase relative permeabilities calculated from two-phase Corey- $\mathrm{k}_{\mathbf{r}}$ and two-phase extended Corey- $\mathrm{k}_{\mathrm{r}}$ are both treated. Only problems with left and right states outside the elliptic regions are considered. MOC solutions with one of the initial states inside the elliptic regions are in theory feasible, but in practice, the small size of the elliptic regions (Guzman and Fayers, 1996) does not justify the extra complications in the construction of such solutions. Solutions with initial left and right states within the hyperbolic region stay within the hyperbolic region (Holden et al. 1990), and the problem can be treated as described previously. It is still necessary to show that MOC solutions for states that lie on opposite sides of an elliptic region give stable shock solutions as indicated by the numerical solutions (Bell et al., 1986).

\section{Solutions With Two-Phase Corey-kr}

This section presents solutions to three-phase Riemann problems using the relative permeabilities of the UKLB data set. The relative permeabilities and the viscosity ratios used $\left(\mu_{o} / \mu_{g}=40\right.$ and $\left.\mu_{o} / \mu_{w}=3.33\right)$ are representative of realistic reservoir and laboratory conditions. Fig. 6 shows the rarefaction curves and elliptic regions for this example. The rarefaction curves are very similar to the rarefaction curves for the unique umbilic point model (Guzman, 1995). This property is not unexpected since the model in Fig. 6 is produced by perturbations in the relative permeabilities of the Corey-type model: A key difference is the appearance of a small fan of 2-rarefaction curves as the umbilic point opens up to create the central elliptic region of Fig. 6. The small elliptic regions near maximum gas and water saturation of Fig. 6 do not seem to have a large influence in the global structure of the rarefactions.

MOC and FD solutions to the Riemann problem representing injection of essentially $100 \%$ gas into a reservoir with $60 \%$ water and $40 \%$ oil is shown in Fig. 7. The injection (left) condition is located on the border of the three-phase mobile region since gas cannot be injected at saturations lower than residual oil and connate water. The MOC solution in Fig. 7 is composed of a 2-rarefaction wave from point $a$ to $b$, and a 2-Buckley-Leverett shock from $b$ to $c$. Point $b$ is on the 2-interior contact locus and is found by solving the equations for the Rankine-Hugoniot and entropy conditions (Eqs. 8 and 9). The upstream and downstream states of the shock are located on opposite sides of the main elliptic region, and as predicted by the numerical solutions of Bell et al. (1986), the shock is not influenced by the presence of the elliptic region. The FD solution is very close to the MOC solution, but still has the same overshooting problem discussed before. The small elliptic region near the point a does not seem to have much influence on the solution.

The effect of gravity can be observed in Fig. 8. The initial conditions are the same as in the example of Fig. 7. Positive gravity numbers of $\mathrm{N}_{\mathrm{g}}=10$ and $\mathrm{N}_{\mathrm{w}}=0.2$ were used. Fig. 8 also shows the location of the elliptic regions for this example. The rarefaction curves are very similar to the non gravity example of Fig. 6. The MOC solution in Fig. 8 is composed of a 2-rarefaction wave from $\mathbf{a}$ to $\mathbf{b}$, an indifferent wave between $\mathbf{b}$ and $\mathbf{c}, \mathbf{a}$ rarefaction wave from $\mathbf{c}$ to $d$ on the two-phase oil-water line, and finally a standard two-phase Buckley-Leverett shock from d to the right state $e$. The solution is constructed following the same procedure as in the solution of Fig. 5. The positive gravity numbers decrease the wave speeds inside the three-phase regions compared to the speeds in the two-phase line $S_{\mathrm{g}}=0$ in such a way that the shock from $b$ to $c$ no longer lands directly at the right state. The shock now lands at point c with equal shock and characteristics velocities upstream and downstream of the shock, creating the indifferent wave between $b$ and $c$. The solution then increases the velocity up to point $\mathbf{d}$ where it then shocks to point e.

\section{Solutions With Two-Phase Extended Corey- $\mathbf{k} \mathbf{r}$}

The experimental data of Saraf and Fatt (1967) were selected to construct the following examples. These data show an unusually larger residual oil saturation to gas than to water $\left(S_{\text {org }}=0.3>S_{\text {orw }}=0.1\right.$ ), and a very small relative permeability to gas at residual oil saturation $\left(\mathrm{k}_{\mathrm{rgro}}=0.07\right)$. Viscosity ratios of $\mu_{o} / \mu_{g}=40$ and $\mu_{o} / \mu_{w}=3.33$ were used.

Fig. 9 shows the rarefaction curves and elliptic regions for this example without gravity. As expected, the rarefaction curves shown in Fig. 9 have similar structure to the rarefaction curves for the two umbilic point model of Fig. 2. The behavior observed near the umbilic points in Fig. 2 is almost reproduced by the rarefaction curves near the elliptic regions of Fig. 9.

An example solution with a shock with states on opposite sides of an elliptic region is shown in Fig. 10. The left state $s_{l}=(0.24,0.47)$ corresponds to almost $100 \%$ gas injection. The initial or right state is at critical gas saturation and $42 \%$ water saturation, i.e., $s_{r}$ $=(0.42,0.05)$. The solution is simply a 2 -rarefaction wave from a to $\mathbf{b}$, followed by a 2 -Buckley-Leverett shock from $b$ to $c$. The displacement efficiency is still low due to the small gas viscosity, but has been improved by the reduction in gas relative permeability, when compared to the displacement examples for the UKLB data set (compare shock velocity of approximately 4 in Fig. 10 to the shock velocities of more than 6 in the solution of Fig. 7). The location of the central elliptic region in Fig. 10 being closer to the maximum gas saturation than in Fig. 7, is an indication of the improved gas displacement efficiency in Fig. 10. 


\section{DISCUSSION}

A mathematical theory for semi-analytical solutions of three-phase flow problems in porous media has been presented. The theory provides a foundation to understand the main mathematical features of threephase flow. Occurrence of elliptic regions in the saturation space did not pose intractable problems or physically unacceptable effects on the solutions. The effects of gravity were included, and three-phase flow displacements in inclined reservoirs and gravity segregation problems can be solved. The theory developed is applicable to gas injection processes used to enhance oil recovery from waterfloods (either as a continuous gas phase or as a mixture of gas and water in WAG processes), thermal recovery, miscible gas injection, surfactant flooding, gas gravity drainage following a period of waterflooding, and waterflooding following a period of gas gravity drainage.

The analysis on the location of the umbilic point(s) and the elliptic regions (Guzman and Fayers, 1996) proved to be very useful in the construction of the solutions. The occurrence and location of the singularities were found to dictate the character of the solutions. A strong relationship between the physical and mathematical properties of three-phase flow was shown. Mathematical confirmation has been given to the physical expectations that efficiency of a displacements was discussed. Physically, the efficiency of a displacement can be increased by increasing the displacing phase viscosity, reducing the displacing phase relative permeability, increasing the gravity number for gas, and decreasing the gravity number for water. It was shown that the increase in displacement efficiency, caused by changes in fluid and flow parameters, can be explained by the movement of the umbilic point(s) and elliptic regions towards the maximum of the displacing phase saturation.

Stable solutions were found for initial conditions on opposite sides of the elliptic regions with shocks connecting states across the elliptic regions. Shocks do not satisfy the partial differential equations, but related integral equations (material balance), and therefore, are not affected by the complex value of the eigenvalues in the elliptic regions. Numerical finite difference solutions are, on the other hand, continuous solutions that can traverse the elliptic regions. However, finite difference solutions solve a modified equation that include higher order terms (primarily diffusion) and it appears that these higher order terms, together with nonlinearities of the equations, make the solutions stable inside the linearly unstable elliptic regions (Bell et al., 1986) This indicates that elliptic regions are of no concern for numerical solutions (unless eigenvalue information is used as in Godunov schemes), or in MOC solutions with initial states outside elliptic regions.

\section{CONCLUSIONS}

1. Method of characteristics solutions to three-phase flow problems for the different types of equations of the new classification (Guzmán and Fayers, 1996) were constructed. Problems with and without gravity were included for these types of equations.

2. Stable shocks with states on opposite sides of elliptic regions were constructed. The presence of elliptic regions did not create intractable problems or physically inadmissible effects on the solutions.

3. Solutions to realistic gas and simultaneous water and gas injection problems were constructed. Experimentally based relative permeability data, and realistic values of gravity numbers and viscosity ratios were used.

4. Benchmark solutions that can be used to test numerical simulations of three-phase flow have been constructed.

5. The effects of varying viscosities, gravity numbers, and relative permeabilities were studied. A link between the physical mechanisms and the mathematical theory was explained.

\section{NOMENCLATURE}

$A \quad$ Jacobian matrix of flux vector $f$

$d s \quad$ right eigenvector of matrix $A$

$f \quad$ flux vector with components $f_{w}$ and $f_{g}$

$f_{w} \quad$ water fractional flow

$f_{g} \quad$ gas fractional flow

$f_{p q} \quad \partial f_{p} / \partial S_{q}(\mathbf{p}, \mathbf{q}=\mathrm{g}, \mathrm{w})$

$k_{r p} \quad$ relative permeability

$N_{w} \quad$ water gravity number

$N_{g} \quad$ gas gravity numbers

$S_{g c} \quad$ critical gas saturation

$S_{\text {org }} \quad$ residual oil saturation to gas

$S_{\text {orm }} \quad$ residual oil saturation

$S_{\text {orw }}$ residual oil saturation to water

$S_{p} \quad$ saturation

$S_{w c} \quad$ connate water saturation

$s \quad$ saturation vector $\left(S_{w}, S_{g}\right)$

$s_{l}, s_{r} \quad$ left $(\mathrm{x}<0)$ and right $(\mathrm{x}>0)$ values of $s$

$s_{-}, s_{+} \quad$ shock up and down-stream values of $s$

$t$ dimensionless time (injected pore volumes)

$x$ dimensionless distance (fraction of length)

\section{Greek letters}

$\delta \quad$ discriminant

$\eta \quad$ eigenvalue, characteristic or wave velocity

$\lambda_{p} \quad$ phase mobility

$\mu_{p} \quad$ phase viscosity

$\sigma \quad$ shock velocity 


\author{
Subscripts \\ g gas \\ o oil \\ p phase \\ $t$ derivative with respect to $t$ \\ w water \\ $\mathrm{x}$ derivative with respect to $\mathrm{X}$
}

\section{Acknowledgments}

We are grateful to professor Khalid Aziz for helpful discussions and comments. Financial support for this work was provided by the Stanford University Petroleum Research Institute Reservoir Simulation Program (SUPRI-B).

\section{References}

1. Aanonsen, S.I.: “Application of Fractional-Flow Theory to 3-Phase, 1-Dimensional Surfactant Flooding," Proceedings of the First European Conference on the Mathematics of Oil Recovery, Cambridge, July, 1989, Cambridge University Press, Cambridge, 1992.

2. Azevedo A.V. and Marchesin, D.: "Multiple Viscous Profile Riemann Solutions in Mixed EllipticHyperbolic Models for Flow in Porous Media," Nonlinear Evolution Equations That Change Type, Keyfitz, B.L. and Shearer, M., Springer-Verlag, New. York, 1990.

3. Bell, J.B., Trangenstein, J.A., and Shubin, G.R.: "Conservation Laws of Mixed Type Describing ThreePhase Flow in Porous Media," SIAM J. Applied Math., (December 1986) 46, No. 6, 1000--1017.

4. Buckley, S.E. and Leverett, M.C.: "Mechanism of Fluid Displacement in Sands," Trans., AIME, (1941) 249, 107--116.

5. Falls, A.H., and Schulte, W.M.: "Theory of ThreeComponent, Three-Phase Displacement in Porous Media," SPERE (August 1992a), 377--384.

6. Falls, A.H., and Schulte, W.M.: "Features of ThreeComponent, Three-Phase Displacement in Porous Media," SPERE (November 1992b), 426-432.

7. Fayers, F.J.: "Extension of Stone's Method 1 and Conditions for Real Characteristics in Three-Phase Flow," SPERE (November 1989), 437--445.

8. Glimm, J.: "The Interaction of Nonlinear Hyperbolic Waves," Comm. Pure and Applied Math., (1988) 41, $569--590$.

9. Gorell, S.B.: "Modeling the Effects of Trapping and Water Altemating Gas (WAG) Injection on Tertiary Miscible Displacements," paper SPE 17340, Sixth
Symposium on Enhanced Oil Recovery, Tulsa, OK, April 17--20, 1988.

10. Grader, A.S., and OMeara Jr., D.J.: "Dynamic Displacement Measurements of Three-Phase Relative Permeabilities Using Three Immiscible Liquids," paper SPE 18293, Annual Technical Conference and Exhibition, Houston, TX, October 2--5, 1988.

11. Guzmán, R.E.: "Mathematics of Three-Phase Flow," PhD dissertation, Stanford University, Stanford, C.A (July 1995).

12. Guzmán, R.E. and Fayers, F.J.: "Mathematical Properties of Three-Phase Flow Equations," Accepted for publication to the SPEJ, 1996.

14. Holden, H.: "On the Riemann Problem for Prototype of a Mixed Type Conservation Law," Comm. on Pure and Applied Math., (1987) 40, 229--264.

15. Holden, H., Holden, L., and Risebro, N.H.: "Some Qualitative Properties of $2 \times 2$ Systems of Conservation Laws of Mixed Type," Nonlinear Evolution Equations That Change Type, Keyfitz, B.L. and Shearer, M., Springer-Verlag, New York, 1990.

16. Holden, L., and Hoegh-Krohn, R.: "A Class of Nonlinear Hyperbolic Conservation Laws," J. Diff. Eqn. (1990) 84, 73--99.

17. Isaacson, E.L., Marchesin, D., and Plohr, B.J.: "The Structure of the Riemann Solution for NonStrictly Hyperbolic Conservation Laws," Second International Conference on Nonlinear Hyperbolic Problems, Aachen, FGR\}, March, 14--18, 1988.

18. Isaacson, E.L., Marchesin, D., and Plohr, B.J.: "Transitional Shock Waves," Contemporary Mathematics (1989) 100, 125--145.

19. Johnson, E.F., Bossler, D.P., and Naumann, V.O.: "Calculation of Relative Permeability from Displacemt Experiments," Trans. AIME (1959) 216, 370.

20. Lax, P.D.: "Hyperbolic Conservation Laws II," Comm. Pure and Applied Math. (1957) 10, 537--566.

21. LeVeque, R.J.: "Numerical Methods for Conservation Laws," second edition, Birkhäuser Verlag, Basel, 1992.

22. Liu, T.P.: "The Riemann Problem for General Systems of Conservation Laws," J. Diff. Eqn. (1975) 18, 218--234.

23. Oleinik, O.A.: "Discontinuous Solutions of NonLinear Differential Equations," Uspekhi Mat. Nauk (N.S) (1957) 12, No. 3 (75), 3-73, English translation in: Amer. Math. Soc. Transl., Ser. 2, v. 26, 95--172, (1963). 
24. Saraf, D.N., and Fath, I.: "Three-Phase Relative Permeability Measurement Using a Nuclear Magnetic Resonance Technique for Estimating Fluid Saturation," JPT, Trans., AIME (September 1967) 240, 235--242.

25. Sahni , A., Guzmán, R.E., and Blunt, M.J.: "Three-Phase Flow: From Analysis of Experiments to Prediction at Field Scale," paper SPE 36664, 71st Annual Technical Conference and Exhibition, Denver, Colorado, Octoher 6-9, 1996.

26. Shalimov, B.V.: "Flow of a Three-Phase Fluid in a Porous Medium (Buckley-Leverett Model)," Izv. Akad. Nauk SSSR, Mekh. Zhidk. Gaza (1972) 39, No. 1, 3944.

27. Stone, H.L.: "Probability Model for Estimating Three-Phase Relative," JPT Trans. AIME (February 1970) 249, 241-218.

28. Virnovsky, G.A.: "Determination of Relative permeabilities in a Three-Phase Flow in a Porous Medium," Izv. Akad. Nauk SSSR, Mekh. Zhidk. Gaza (1984) 5, 187-189.

29. Wingard, J.S. and Orr, F.M., Jr.: "An Analytical Solution for Steam/Oil/Water Displacements," SPE Advanced Technology Series (1994) 2, No. 2, 167-176.

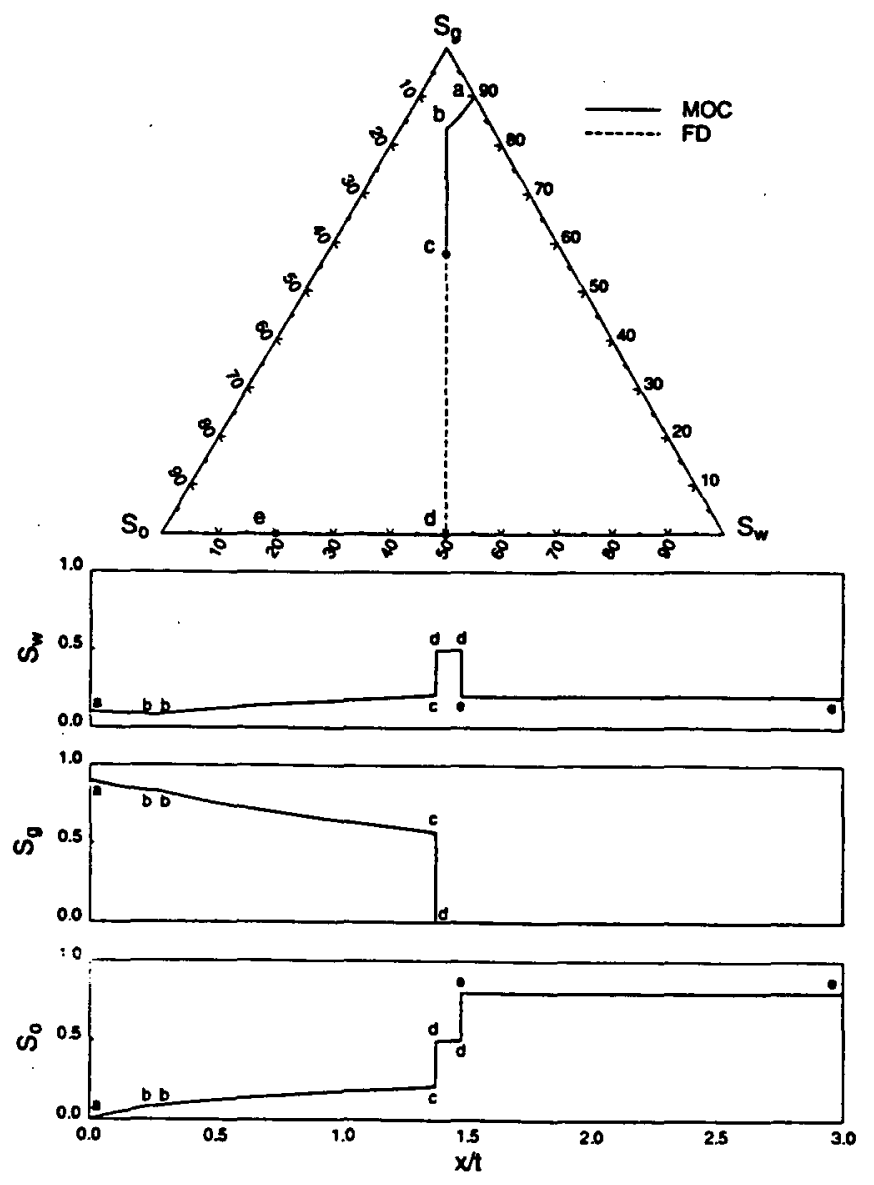

Figure 1. MOC. and FD solutions for symmetric exar.ple, $s_{l}=(0.1,0.9)$, and $s_{r}=(0.2,0)$.

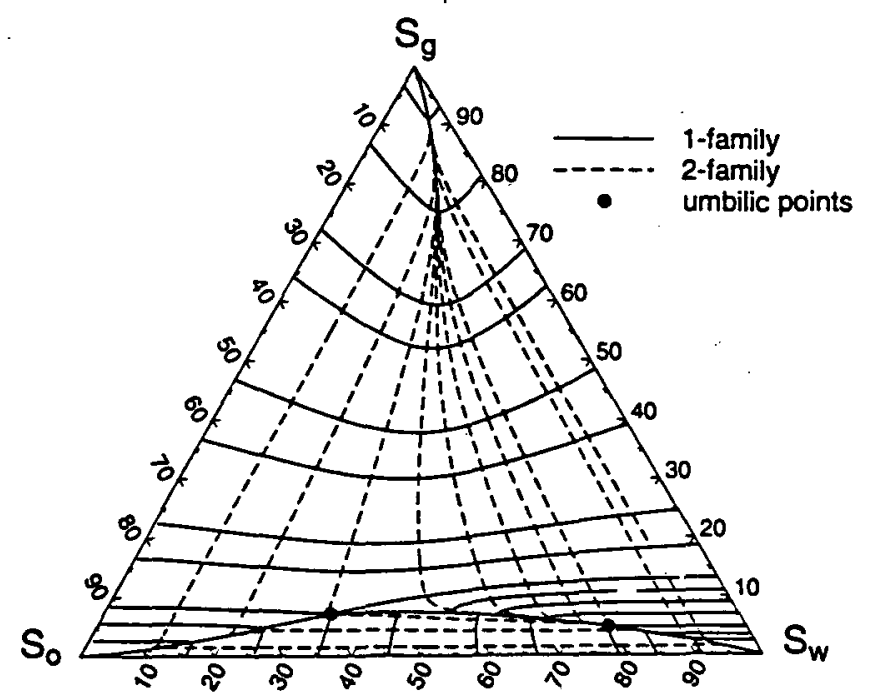

Figure 2. Rarefaction curves for example with two isolated interior umbilic points.

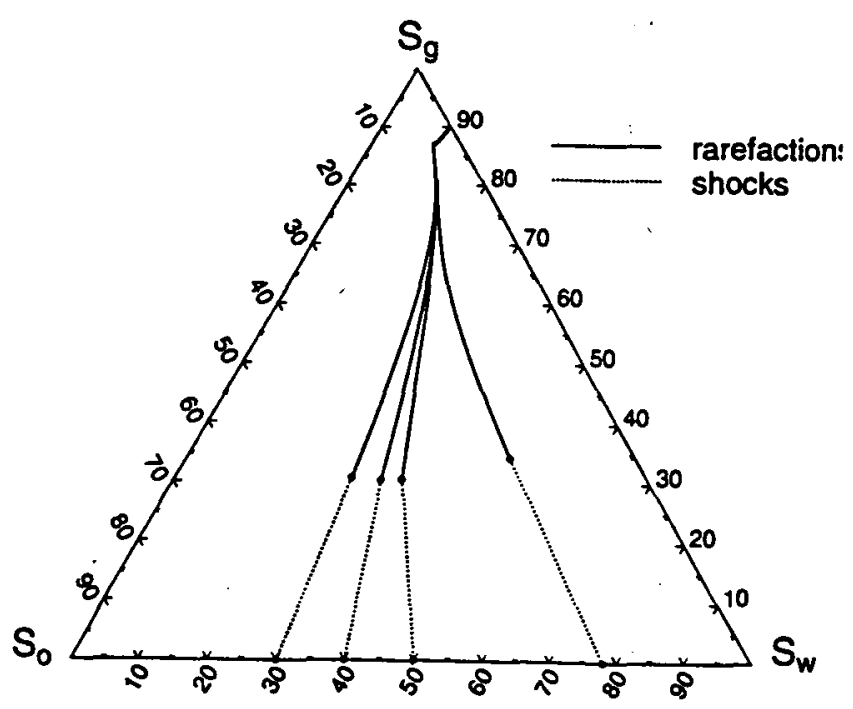

Figure 3. MOC solutions for examples with the sane left state and four different right states. Extended Corey$\mathbf{k}_{\mathbf{r}}$ yielding two-umbilic points. 

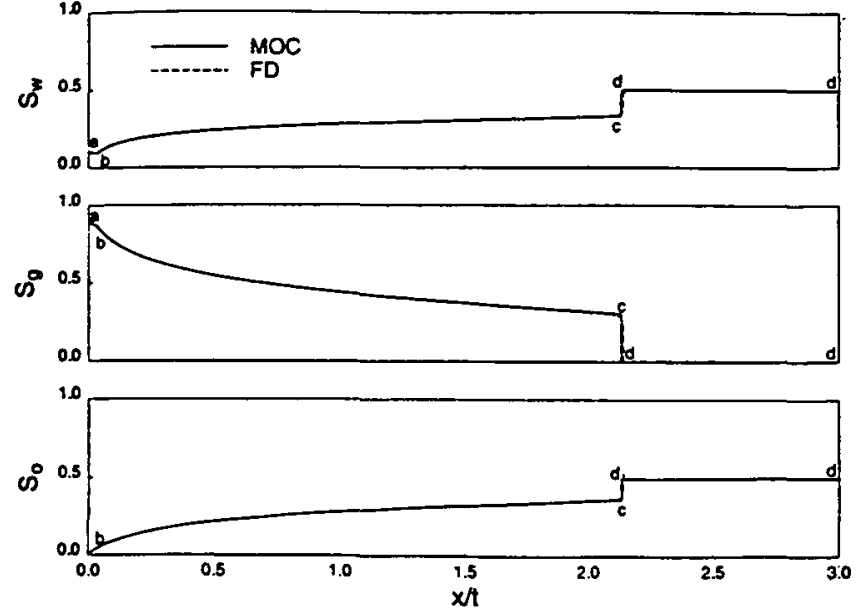

Figure 4. MOC. and FD saturation profiles for $s_{l}=$ $(0.1,0.9), s_{r}=(0.5,0)$. Extended Corey- $\mathrm{k}_{\mathrm{r}}$ yielding twoumbilic points.
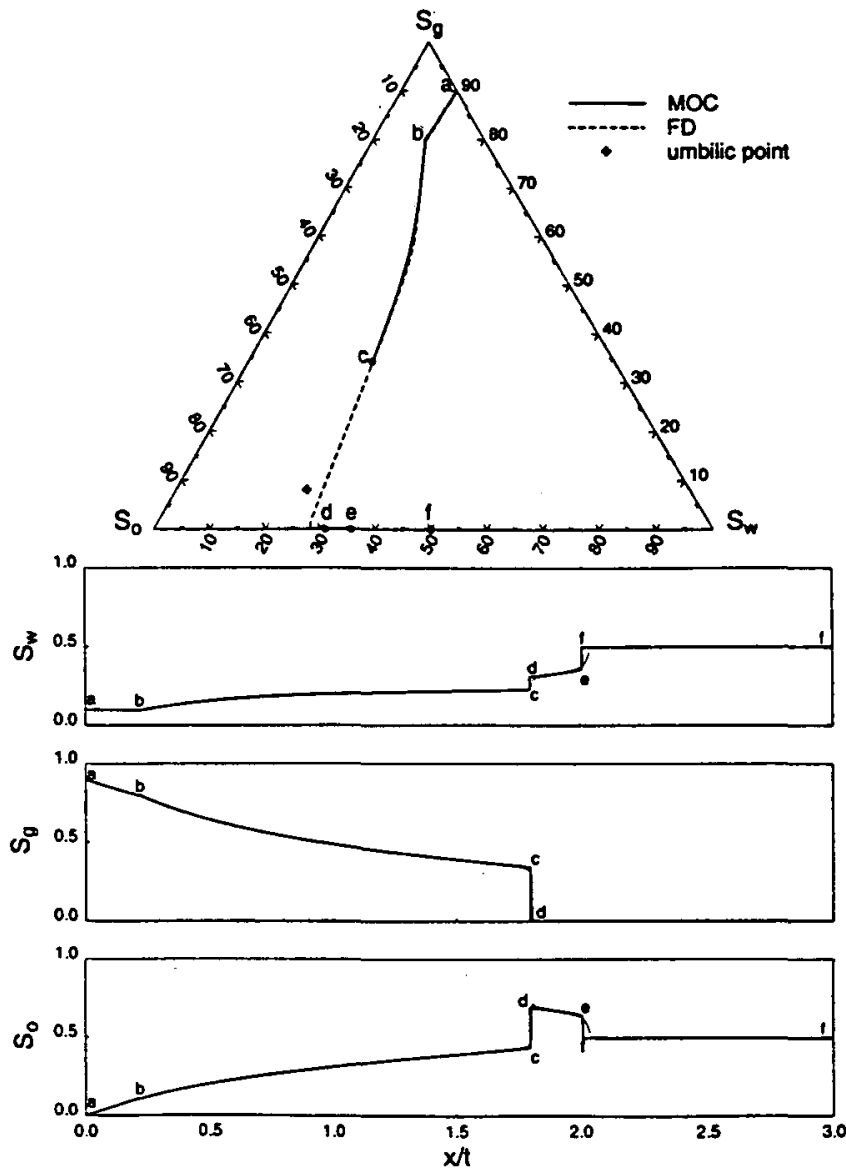

Figure 5. MOC. and FD solutions for $s_{l}=(0.1,0.9)$, $s_{r}=(0.5,0), N_{g}=2$. and $N_{W}=1 / 2$. Extended Corey $-k_{r}$ yielding one umbilic point.

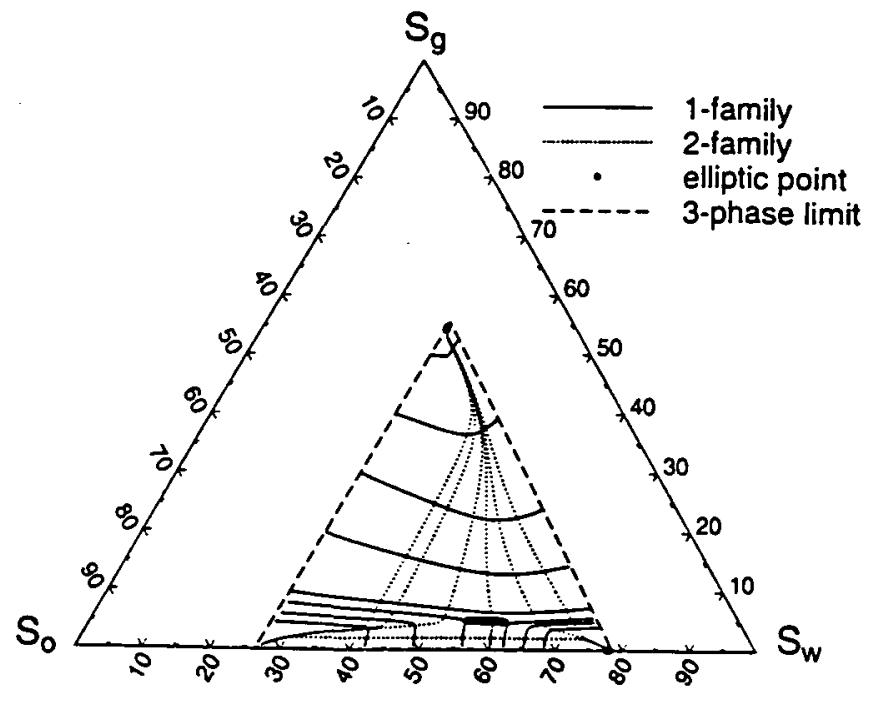

Figure 6. Rarefaction curves for the UKLB data set without gravity.

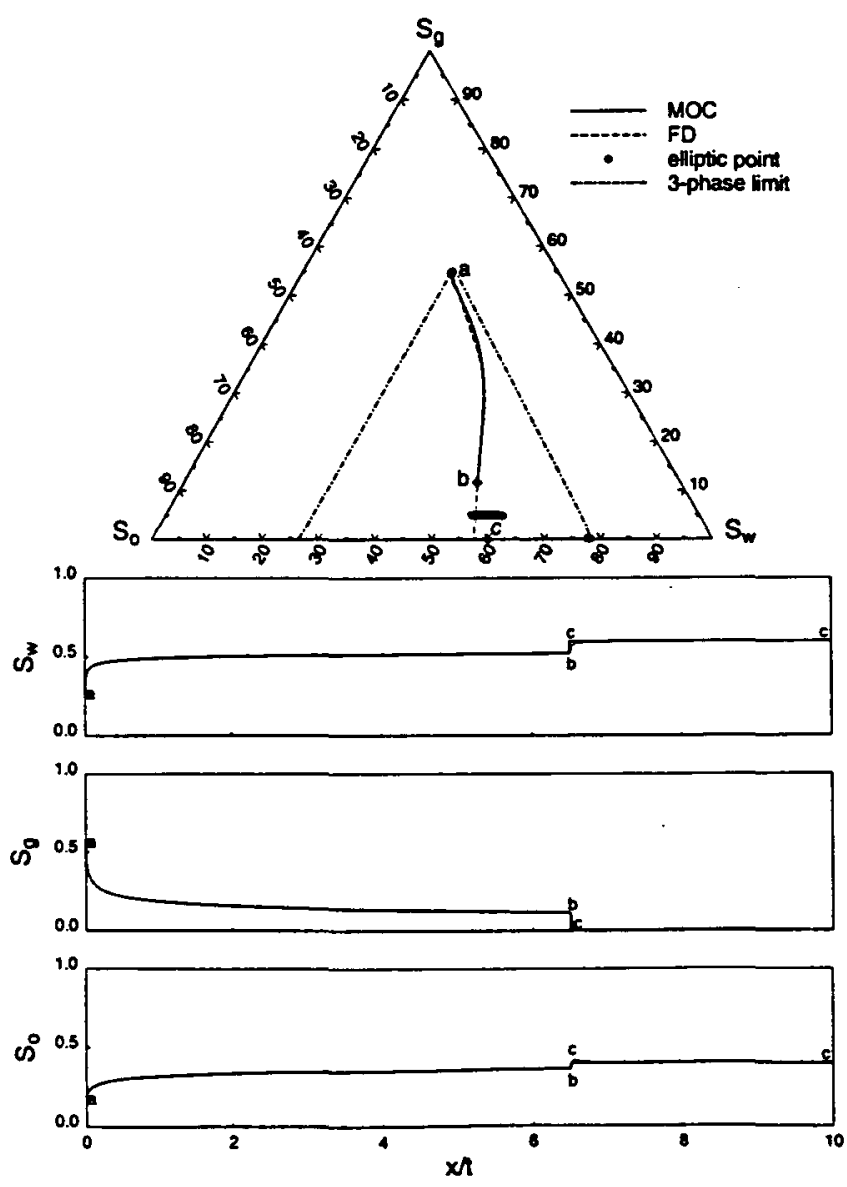

Figure 7. MOC and FD solutions for the UKLB data set with $s_{l}=(0.27,0.54)$ and $s_{r}=(0.6,0)$. A stable shock with states on opposite sides of elliptic region is shown. 


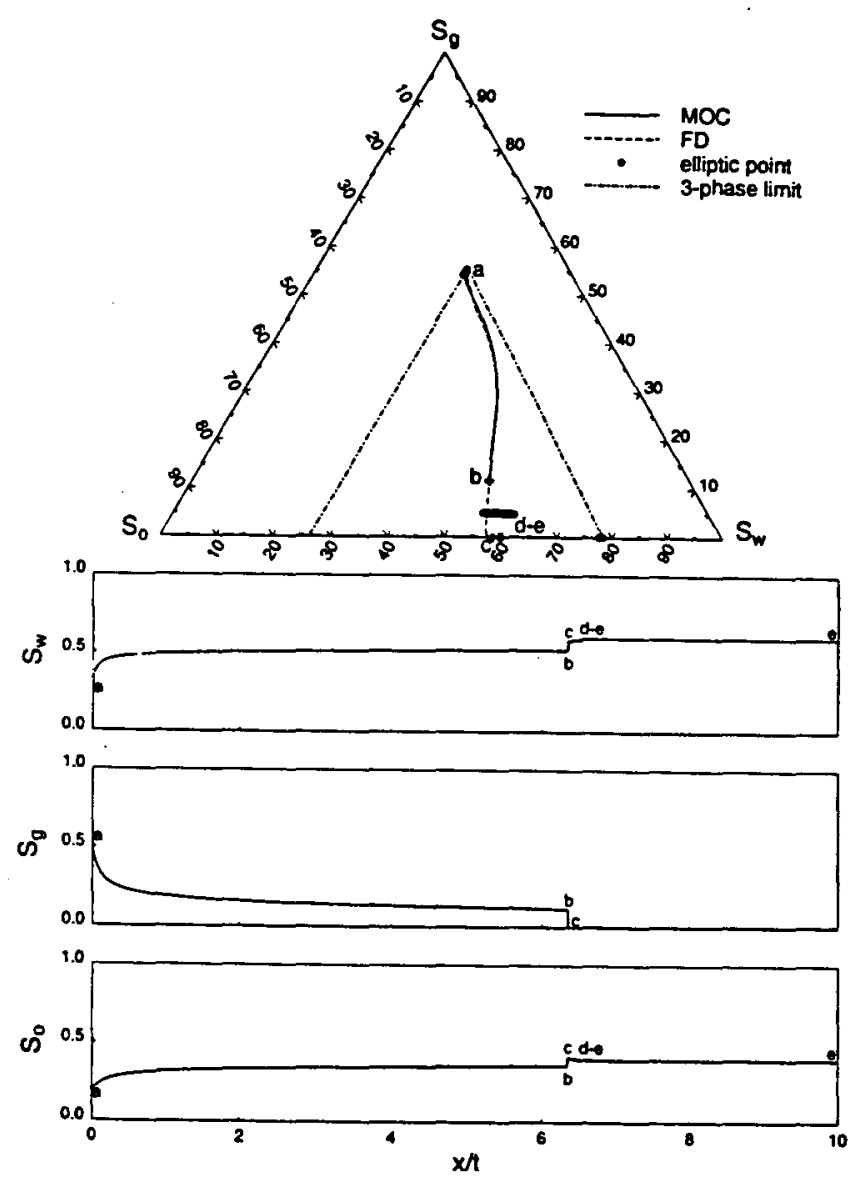

Figure 8. MOC and FD solutions for the UKLB data set with $s_{l}=(0.27,0.54), s_{r}=(0.6,0), \mathrm{N}_{\mathrm{g}}=10$, and $\mathrm{N}_{\mathrm{W}}=$ 0.2 .

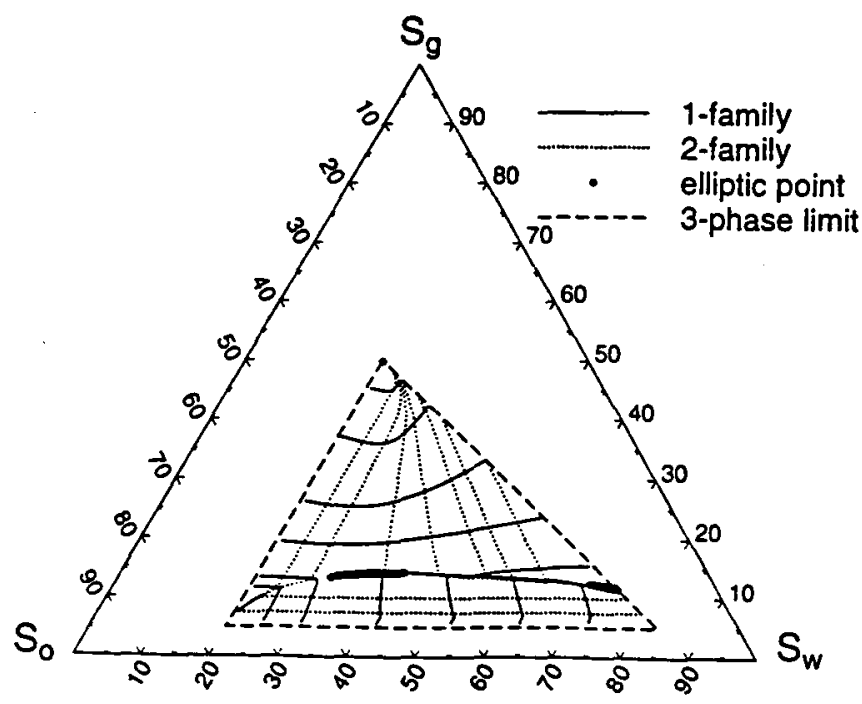

Figure 9. Rarefaction curves for the Sarat and Fatt (1967) data set wilhout gravity.

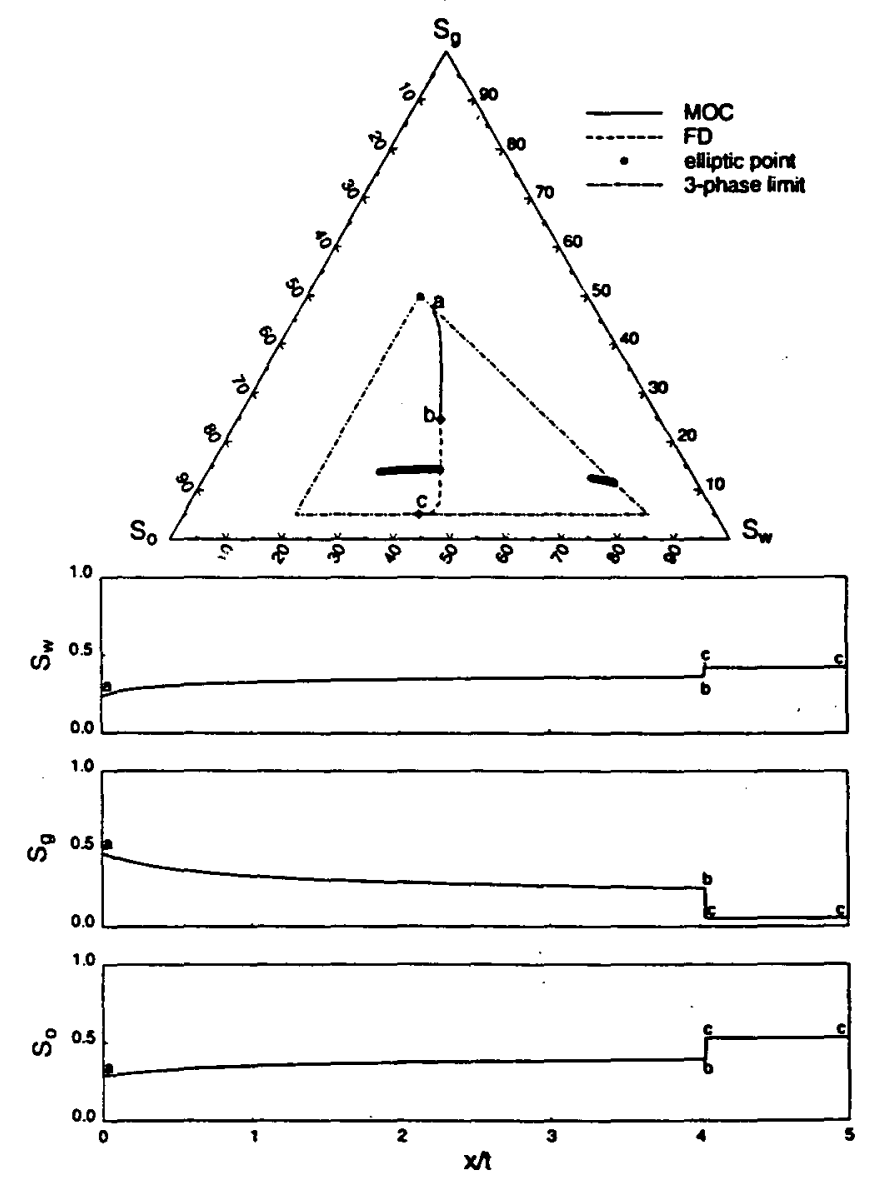

Figure 10. MOC and FD solutions for the Saraf and Fatt $(1967)$ data set with $s_{l}=(0.24,0.47)$ and $s_{r}=$ $(0.42,0.05)$. Stable shock with states on opposite of an elliptic region. 